Article

\title{
A Magneto-Hyperelastic Model for Silicone Rubber-Based Isotropic Magnetorheological Elastomer under Quasi-Static Compressive Loading
}

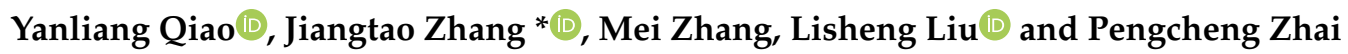 \\ Hubei Key Laboratory of Theory and Application of Advanced Materials Mechanics, School of Science, \\ Wuhan University of Technology, Wuhan 430070, China; QiaoYanliang@whut.edu.cn (Y.Q.); \\ zhangmei@whut.edu.cn (M.Z.); liulish@whut.edu.cn (L.L.); pczhai@126.com (P.Z.) \\ * Correspondence: zhjiangtao@whut.edu.cn; Tel.: +86-27-8765-1820
}

Received: 29 September 2020; Accepted: 20 October 2020; Published: 22 October 2020

check for updates

\begin{abstract}
A new magneto-hyperelastic model was developed to describe the quasi-static compression behavior of silicone rubber-based isotropic magnetorheological elastomer (MRE) in this work. The magnetization property of MRE was characterized by a vibrating sample magnetometer (VSM), and the quasi-static compression property under different magnetic fields was tested by using a universal testing machine equipped with a magnetic field accessory. Experimental results suggested that the stiffness of the isotropic MRE increased with the magnetic flux density within the tested range. Based on experimental results, a new magneto-hyperelastic model was established by coupling the Ogden hyperelastic model, the magnetization model and the magneto-induced modulus model based on a magnetic dipole theory. The results show that the proposed new model can accurately predict the quasi-static compression property of the isotropic MRE under the tested magnetic flux density and strain ranges using only three model parameters.
\end{abstract}

Keywords: magnetorheological elastomer; magnetorheological effect; quasi-static compression; magneto-hyperelastic model

\section{Introduction}

Magnetorheological elastomer (MRE) is a kind of smart material composed of micron-sized ferromagnetic particles dispersed in a polymer matrix [1-3], which is often referred to as a solid-state analogue of magnetorheological fluid (MRF) [4,5]. It has been shown that the mechanical properties of MRE could be rapidly and reversibly controlled by the external magnetic field due to magnetic dipole-dipole attractions between the magnetized particles [1]. This unique magnetic-field-dependent property makes MRE have numerous potential engineering applications, such as vibration absorber [6], vibration isolator [7], sensing device [8] and other smart MRE devices [9,10].

With the increasing application of MRE in compression mode, both experimental analysis and theoretical modeling are required to predict the response of MRE under quasi-static compressive loading, thus advancing the development of MRE devices. Previous experimental studies have shown that the mechanical responses of MRE are strongly nonlinear functions of magnetic flux density and compressive loading $[3,11]$, which makes it still a substantial challenge to develop constitutive models to describe MRE behaviors. In general, there are two very notable approaches to describe the stress-strain response of MRE under magneto-mechanical coupling conditions, which are microscopic approaches based on the magnetic dipole approximation [1,2,12,13] and macroscopic approaches based on the experimental phenomena [3,14-16].

The microscopic dipole approach is generally carried out based on the spatial distribution hypothesis of particles in MRE, and each particle is assumed as a magnetic dipole [1,2]. For example, 
Jolly et al. [1] developed a simple dipole model in their early work, which was based on the magnetic interactions between two adjacent particles to analyze the MRE properties. Davis [2] proposed another dipole model to predict the magneto-induced modulus by assuming that the particles form an infinite chain in the matrix. Ivaneyko et al. [12] extended the previous approaches and proposed three different lattice models to represent the particle distribution in the MRE. In their works, the magneto-induced deformation and Young's modulus of MRE were calculated as functions of the external magnetic field strength. Liao et al. [13] proposed a modified magnetic dipole model to investigate the evolution of the normal force of MRE with particle magnetization under the compression state. As found from the above works, magnetic dipole theory microscopically provided some fundamental bases for describing the relationship between the microstructure and mechanical properties of MRE [17]. Unfortunately, the lack of consideration of the reconstruction of particle distribution during MRE deformation makes it difficult to analyze the response of MRE at complex loads, such as large deformations.

For the macroscopic phenomenological approach, previous works mainly focused on developing magneto-mechanical coupling models by fitting experimental results. Varga et al. [3] proposed a phenomenological approach to describe the quasi-static compression property of MRE, in which the magneto-induced elastic modulus was calculated as a function of magnetic flux density. Koo et al. [14] developed a model to capture the compression behaviors of the MRE under cyclic loading by using a multi-layer perceptual artificial neural network. Liao et al. [15] proposed a magneto-mechanical coupling model consisting of hyperelastic, viscoelastic and magnetic components to describe the compression property of MRE under high strain rate. These phenomenological models can be used to predict the MRE behavior under complex loading conditions, even to solve the frequency- and strain-amplitude-dependent problems [16]. However, none of those models had strict microscopic justification or established the explicit functional relationship between the model parameters and the magnetic property [18]. Therefore, it is necessary to develop new phenomenological models to predict the magneto-mechanical coupling behaviors of MRE, which considers such magnetic property and the magnetic field dependence of model parameters.

In this work, a new magneto-hyperelastic model was developed to describe the quasi-static compression property of the isotropic MRE under different magnetic fields. Firstly, a silicone rubber-based isotropic MRE with a particle content of $60 \mathrm{wt} . \%$ was prepared, and the magnetization property was tested. The magnetization property of the MRE was described by an empirical model. Then, the quasi-static compression property of the MRE was experimentally tested and modeled using a first-order Ogden model, and a magnetic-field-dependent function of the stiffness parameter in the Ogden model was proposed based on a magnetic dipole model. Combined with the empirical magnetization model, a magneto-hyperelastic compression model of the isotropic MRE as a function of strain and magnetic flux density was finally developed, which was verified by comparing the model predictions with experimental data.

\section{Materials and Experiments}

\subsection{Materials Preparation}

The isotropic MRE used in this paper was prepared by a two-component liquid silicon rubber (type HY-E630, from Shenzhen Hong Ye Jie Technology Co., Ltd., Shenzhen, China) and carbonyl iron particles (CIPs) with an average diameter of $2 \mu \mathrm{m}$ (type HD-R-1, from Shaanxi Xu Li Heng New Material Co., Ltd., Shaanxi, China). According to the CIP content (60 wt.\%) used in MRE and the manufacturer's recommended weight ratio of the two components (1:1) of silicon rubber, component $A$, component $\mathrm{B}$ and $\mathrm{CIPs}\left(\mathrm{A}_{\text {weight }}: \mathrm{B}_{\text {weight }}: \mathrm{CIPs}\right.$ weight $\left.=1: 1: 3\right)$ were weighed, respectively. Then, the weighed CIPs were divided into two parts equally and mechanically mixed with component $A$ and component $B$ of silicon rubber for $10 \mathrm{~min}$, respectively. Thus, the CIPs were uniformly dispersed in the two liquid silicon rubber components. After that, the two suspensions were blended together and mechanically mixed for $5 \mathrm{~min}$ at the recommended weight ratio of 1:1. The final mixture was poured into the 
aluminum molds ( $\varphi 9 \mathrm{~mm} \times 10 \mathrm{~mm}$ ) and then degassed in a vacuum chamber for $10 \mathrm{~min}$ followed by curing at $50^{\circ} \mathrm{C}$ for $10 \mathrm{~h}$. After that, the isotropic MRE samples were obtained.

\subsection{Microstructure, Magnetization Characterization and Compression Experiments}

The microstructure of the prepared MRE samples was characterized by scanning electron microscope (SEM) (JSM-7500F from JEOL Ltd., Tokyo, Japan). The sample was first cryogenically fractured in liquid nitrogen and then dried in a vacuum. The fractured surface was coated with carbon powders for SEM observation, and the scanning voltage was set at $5 \mathrm{kV}$.

The magnetization property of the prepared MRE material in the magnetic field range of 0-1000 mT was tested by using a vibrating sample magnetometer (VSM) (Versalab from Quantum Design Inc., San Diego, CA, USA).

The compression tests of MRE samples under different magnetic fields were performed using a universal testing machine (Instron2382 from Instron Corporation, Norwood, MA, USA) equipped with a magnetic field accessory, i.e., a test fixture with two fixed permanent magnets, as illustrated in Figure 1 . The test fixture was connected to the testing machine via two shafts. Two fixed neodymium permanent magnets $(\varphi 40 \mathrm{~mm} \times 20 \mathrm{~mm}$ ) were used to generate the desired magnetic field with the direction parallel to the axis of the drive shafts. All other parts of the test fixture were made of non-magnetic aluminum alloy with sufficient stiffness. Before each test, the magnetometer probe was placed at the center of the test space, and the magnetic flux density was adjusted by changing the distance between the two magnets. It should be noted that the distance between the magnets remained fixed throughout each test, ensuring a constant magnetic field applied to the samples along the thickness direction.

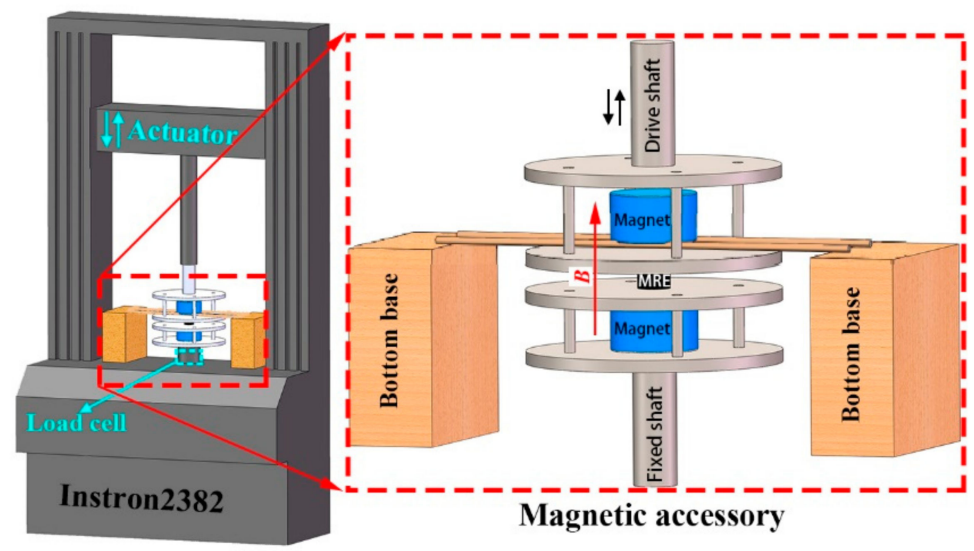

Figure 1. Compression experimental setup.

The compression tests of MRE samples under the magnetic flux densities of $0 \mathrm{mT}, 160 \mathrm{mT}, 275 \mathrm{mT}$ and $330 \mathrm{mT}$ were tested. The compression speed was set at $0.6 \mathrm{~mm} / \mathrm{min}$, and the corresponding initial strain rate was $1 \times 10^{-3} / \mathrm{s}$. The samples were compressed to the maximum strain of $30 \%$. To minimize the effect of the friction between the samples and the fixtures, silicone oil was applied to the two ends of the samples during the compression tests. For each magnetic flux density, three measurements were carried out, and the results were averaged based on three measurements.

\section{Experimental Results and Modeling}

\subsection{Microstructure}

The SEM image of prepared MRE material is shown in Figure 2. It can be seen that spherical CIPs were uniformly distributed in the matrix, and almost no porosity was observed in the sample. This microstructure suggests that a dense and homogeneous MRE material was obtained in this work. 


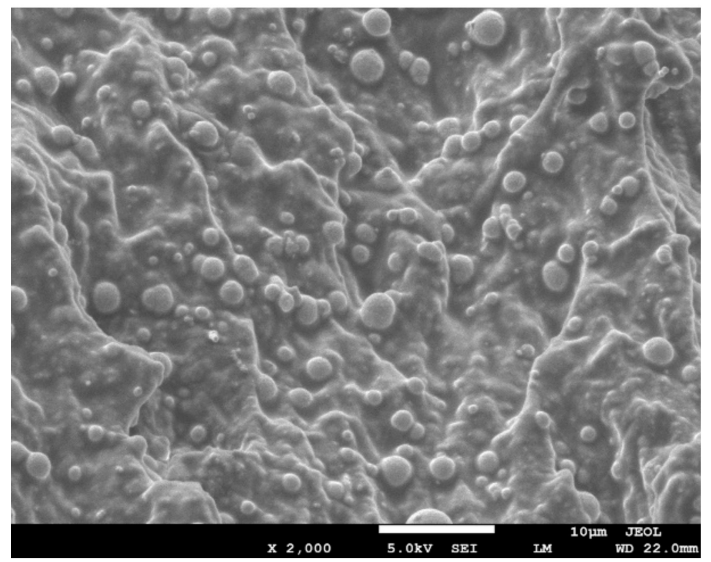

Figure 2. SEM image of the prepared isotropic magnetorheological elastomer (MRE) material.

\subsection{Magnetization Property and Magnetization Model}

The magnetization curve of the prepared MRE is illustrated in Figure 3. It can be seen that the prepared MRE sample showed the typical magnetization characteristics of soft magnetic material. According to the empirical model proposed by Liu et al. [19], the relationship between the magnetization and the magnetic flux density $B$ can be expressed as

$$
M_{M R E}(B)=M_{S}\left(1-e^{-\chi\left(B / \mu_{0}\right)}\right)
$$

where $M_{M R E}$ and $M_{S}$ are the magnetization and saturation magnetization of the MRE material, respectively, $\chi$ is the adaptive magnetization coefficient, $B$ is the magnetic flux density of the external magnetic field and $\mu_{0}=4 \pi \times 10^{-7} \mathrm{H} / \mathrm{m}$ is the permeability of vacuum.

Equation (1) is used to fit the magnetization curve of the MRE, and the fitting parameters are as follows: $M_{S}=307.6 \mathrm{kA} / \mathrm{m}$ and $\chi=3.01 \times 10^{-6} \mathrm{~m} / \mathrm{A}$. The comparison of the fitted results with the experimental data is shown in Figure 3. The precision of the fitting result was quantitatively evaluated by using the coefficient of determination $R^{2}$ [20], which is defined by

$$
R^{2}=1-\sum\left(\Gamma_{\exp }-\Gamma_{\bmod }\right)^{2} / \sum\left(\Gamma_{\exp }-\bar{\Gamma}_{\exp }\right)^{2}
$$

where $\Gamma_{\exp }$ and $\Gamma_{\text {mod }}$ are the experimental data and fitting results, respectively. $\bar{\Gamma}_{\text {exp }}$ is the average value of the experimental data. When the coefficient of determination $R^{2}$ is equal to 1 , it indicates that the results calculated by the model are exactly the same as the experimental data. Here, the value of $R^{2}$ was 0.9044 in the magnetic field range of 0-1000 mT. However, in the magnetic field range of 0-330 mT, in which the compressive tests of MRE were performed, the value of $R^{2}$ was 0.9989 .

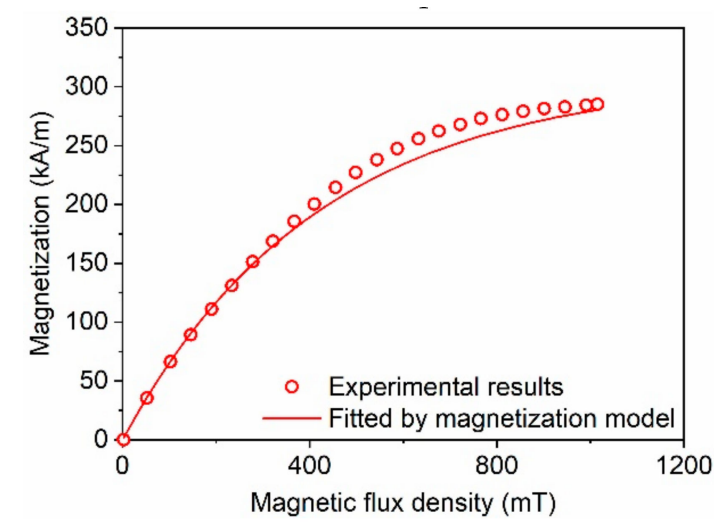

Figure 3. Comparison of the magnetization curves between model calculation and experimental test. 


\subsection{Compression Experimental Results}

The quasi-static compressive stress-strain curves of the isotropic MRE under different magnetic flux densities are shown in Figure 4, in which the compressive stress and strain are defined as negative values, and the experimental results are plotted by the color hollow dots. It was found that at a given strain, the compression stress increased with the increasing magnetic flux density. This phenomenon is called the magnetorheological (MR) effect of MRE, which is caused by the magnetic attractions between magnetized particles in MRE under the external magnetic field [1,2]. When applying an external magnetic field, the interparticle magnetic attractions will lead to a three-dimensional network structure of magnetic particles in the MRE, which serves as a reinforcing frame and offers restriction to the mobility of the polymer chains, thus improving the stiffness of MRE under the compressive loading.

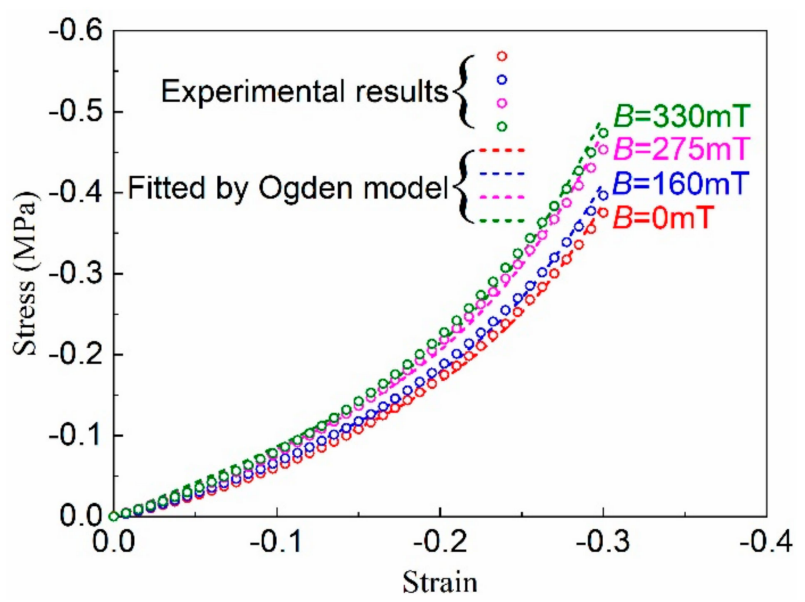

Figure 4. Quasi-static compressive stress-strain curves of the isotropic MRE under different magnetic flux densities.

As shown in Figure 4, the maximum compressive stress under zero magnetic field was $0.38 \mathrm{MPa}$ at the strain of 0.3 , and it was $0.48 \mathrm{MPa}$ under the magnetic flux density of $330 \mathrm{mT}$. The absolute and percentage increments were $0.10 \mathrm{MPa}$ and $26.3 \%$, respectively, representing the absolute MR effect and the relative MR effect [21]. It could be also seen that the increasing trend of the stress with the strain was basically the same under different magnetic fields, indicating that the strain hardening of MRE under different magnetic fields was controlled by that of the rubber matrix.

\subsection{Magneto-Hyperelastic Model}

In the following sections, the compressive stress-strain curves of MRE material under different magnetic flux densities were first fitted by the Ogden hyperelastic model, and the magnetic field dependence of the stiffness parameter in Ogden model was analyzed. Then, a magnetic field-dependent function of the stiffness parameter was proposed based on a magnetic dipole model. Considering the magnetization model, the magneto-hyperelastic model as a function of strain and magnetic flux density was developed, which was verified by comparing the model predictions with experimental data.

\subsubsection{Ogden Hyperelastic Model}

According to the strain hardening property of the tested MRE material, the Ogden hyperelastic model is used to fit the compressive stress-strain curves [22-24]. The strain-energy function of Ogden model is expressed as the function of the principal stretches $\lambda_{1}, \lambda_{2}$ and $\lambda_{3}$, as shown in the following equation [23]:

$$
W=\sum_{n=1}^{N} \frac{\mu_{n}}{\alpha_{n}}\left(\lambda_{1}^{\alpha_{n}}+\lambda_{2}^{\alpha_{n}}+\lambda_{3}^{\alpha_{n}}-3\right)
$$


where $N$ is a positive integer, $\mu_{n}$ and $\alpha_{n}$ are material parameters, and the shear modulus of the material can be calculated by $\mu=\sum_{n=1}^{N} \mu_{n} \alpha_{n}$. Under the simple uniaxial loading, the axial stress $\sigma$ is given by the following [25]:

$$
\sigma=\sum_{n=1}^{N} \mu_{n}\left(\lambda^{\alpha_{n}-1}-\lambda^{-\left(0.5 \alpha_{n}+1\right)}\right)
$$

where $\lambda$ is the stretch in the loading direction and $\lambda=1+\varepsilon$. As shown in Equation (4), $\alpha_{n}$ determines the strain-hardening characteristic of the materials.

Since the stress-strain curves under different magnetic flux densities show the same strain-hardening trend, here we assumed that the strain-hardening parameter $\alpha_{n}$ is independent of the magnetic field, and it is determined firstly by fitting the Ogden model (Equation (4)) to the stress-strain curve of MRE under the zero magnetic field. It was found that the first-order Ogden $(N=1)$ model was sufficient to obtain an excellent correlation with experimental data. Then, using the determined $\alpha_{1}$, the material parameter $\mu_{1}$ was determined by fitting the first-order Ogden model to the stress-strain curves under different magnetic flux densities. The fitted parameter $\alpha_{1}$, the average value of $\mu_{1}$ and its standard deviation $\pm \Delta \mu_{1}$ are listed in Table 1 . For comparison, the results fitted by the first-order Ogden $(N=1)$ model are also plotted in Figure 4, as illustrated in Section 3.3. The fitted results agreed well with the experimental results. It can be seen from Table 1 that the stiffness parameter $\mu_{1}$ increased with the increase of magnetic flux density, reflecting the MR effect of the isotropic MRE material.

Table 1. The fitted model parameters under different magnetic flux densities.

\begin{tabular}{cccc}
\hline $\boldsymbol{B}(\mathbf{m T})$ & $\boldsymbol{\mu}_{\mathbf{1}} \mathbf{( P a )}$ & $\pm \Delta \boldsymbol{\mu}_{\mathbf{1}} \mathbf{( P a )}$ & $\boldsymbol{\alpha}_{\mathbf{1}}$ \\
\hline 0 & 44,705 & 524 & 10.13 \\
160 & 47,869 & 864 & 10.13 \\
275 & 55,045 & 869 & 10.13 \\
330 & 57,431 & 291 & 10.13 \\
\hline
\end{tabular}

\subsubsection{Magnetic-Field-Dependent Function of Stiffness Parameter}

Since the stiffness parameter $\mu_{1}$ is the only magnetic-field-dependent parameter in the Ogden model, a magnetic-field-dependent function of $\mu_{1}$ is needed for the development of a magneto-hyperelastic model for MRE. This is achieved by analyzing the magnetic potential energy of magnetic particles in MRE under the magnetic field. Based on the magnetic dipole model, Ivaneyko et al. [26,27] obtained the magnetic potential energy of magnetic particles in isotropic MRE as a function of the strain $\varepsilon$, which can be expressed by

$$
U(\varepsilon)=\frac{\mu_{0}}{4 \pi}(\phi M)^{2} f(\varepsilon)
$$

where $\phi$ and $M$ are the volume content and magnetization of CIPs, respectively. Note, that $U(\varepsilon)$ in Equation (5) depends on $\phi$ and $M$ through their combination $\phi M$, which is the magnetization of MRE material $M_{M R E}(B)=\phi M$ [27]. The magneto-induced modulus $\Delta E_{m}$ for an MRE under the magnetic field then can be obtained as the second derivative of magnetic potential energy to $\varepsilon$ as follows:

$$
\Delta E_{m}=\frac{\mu_{0}}{4 \pi} \frac{\partial^{2} f(\varepsilon)}{\partial \varepsilon^{2}} M_{M R E}^{2}(B)
$$

Equation (6) shows that $\Delta E_{m}$ is quadratically proportional to $M_{M R E}$, and the proportionality coefficient is a function of $\varepsilon$. Due to the linear relationship between the material stiffness and modulus, 
the increment of stiffness parameter $\mu_{1}$ of the Ogden model can also be assumed to be quadratically proportional to the magnetization of MRE and is given by

$$
\Delta \mu_{1}(B)=K M_{M R E}^{2}(B)
$$

where $K$ is the material constant and assumed to be independent of $\varepsilon$ for simplicity. It reflects the dependence of the stiffness parameter on the magnetization of MRE. Thus, the magnetic-field-dependent function of $\mu_{1}$ can be defined as

$$
\mu_{1}(B)=\mu_{1}^{0}+K M_{M R E}^{2}(B)
$$

where $\mu_{1}^{0}$ is the stiffness parameter of the first-order Ogden model under zero magnetic field.

Combined with Equation (1), Equation (8) was used to fit the data listed in Table 1 by the least mean square (LMS) algorithm, and the obtained value of parameter $K$ was 0.45 . The comparison between the calculated results by Equation (8) and the fitted parameters listed in Table 1 is shown in Figure 5, in which the standard deviations of $\mu_{1}$ are also plotted as the error bars. The coefficient of determination $R^{2}$ of Equation (8) was 0.9743 , indicating that the values of $\mu_{1}$ could be accurately calculated by Equation (8).

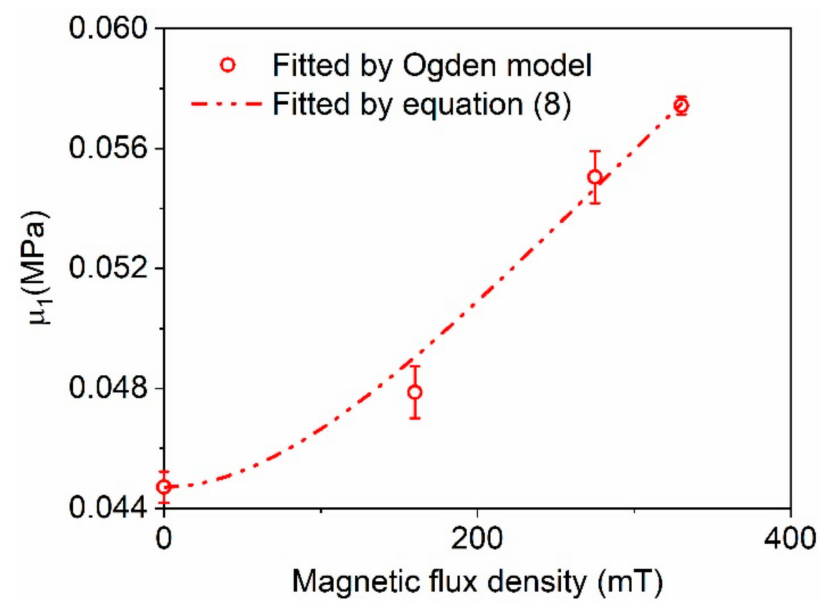

Figure 5. Comparison between the fitting values of stiffness parameter $\mu_{1}$ and the calculation results using Equation (8).

\subsubsection{Magneto-Hyperelastic Model and Discussion}

Substituting Equation (8) into the first-Ogden model, the magneto-hyperelastic constitutive model is finally obtained as follows:

$$
\sigma(\varepsilon, B)=\left(\mu_{1}^{0}+K M_{M R E}^{2}(B)\right)\left((1+\varepsilon)^{\alpha_{1}-1}-(1+\varepsilon)^{-\left(0.5 \alpha_{1}+1\right)}\right)
$$

This model has only three parameters, which were determined in the above sections as $K=0.45$, $\alpha_{1}=10.13$ and $\mu_{1}^{0}=44,705 \mathrm{~Pa}$. The comparison of the compressive stress-strain curves calculated by Equation (9) with the experimental results is shown in Figure 6a. The coefficients of determination $R^{2}$ of the model calculations are $0.9977,0.9976,0.9972$ and 0.9971 under the magnetic flux densities of $0 \mathrm{mT}, 160 \mathrm{mT}, 275 \mathrm{mT}$ and $330 \mathrm{mT}$, respectively. This indicates that the proposed magneto-hyperelastic constitutive model could predict the quasi-static compression property of the prepared isotropic MRE accurately using only three parameters within the tested magnetic flux density and strain ranges. However, it can also be seen from the inserted figure in Figure 6a that the proposed magnetohyperelastic model overestimated the stresses for the strain close to 0.3 under different magnetic fields. This phenomenon is more clearly shown in Figure 6b, in which the predicted compressive stresses as a function of the magnetic flux density were compared with the experimental results under different given strains. This is because the microstructure changed, such as the debonding of the particle/matrix 
interfaces, and the reconstruction of particle distribution happened within MRE material under large deformation, causing the decrease of load-bearing ability and the change of the MR effect of the MRE material $[17,28]$. However, the effects of the microstructure changes were not considered in the present model. In fact, in view of potential practical applications, the strains of MRE material in smart devices usually do not exceed 30\% [29].

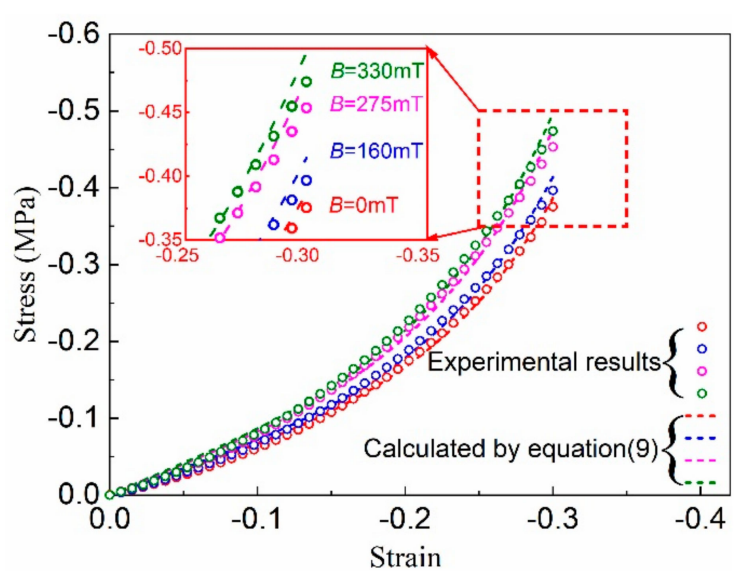

(a)

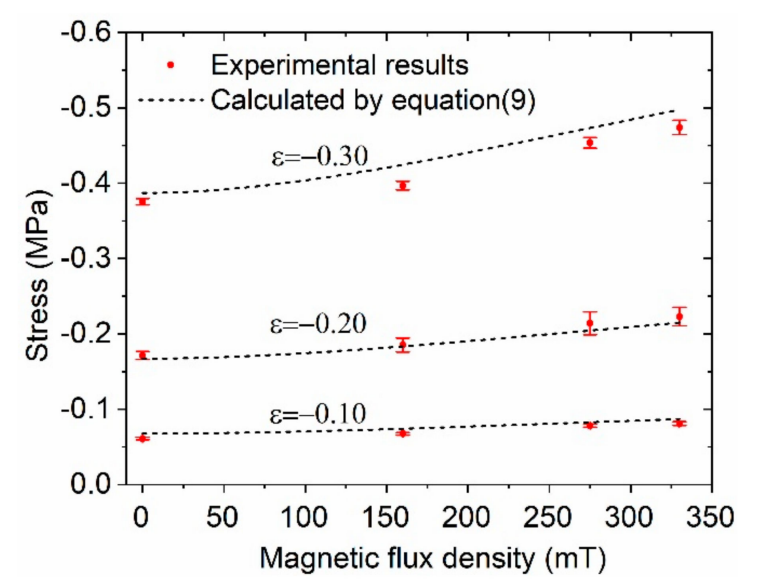

(b)

Figure 6. Comparison of the stresses calculated by the magneto-hyperelastic model (Equation (9)) with experimental results: (a) stress-strain curves under different magnetic flux densities; (b) stress as a function of the magnetic flux density at different strains.

\section{Conclusions}

In this paper, the magnetization property of a silicone rubber-based isotropic MRE and its compression property under different magnetic fields were experimentally tested at first. It was found that the prepared MRE showed a nonlinear magnetization property and an obvious MR effect. Under the magnetic flux density of $330 \mathrm{mT}$ and the compressive strain of $30 \%$, the absolute and relative MR effects were $0.10 \mathrm{MPa}$ and $26 \%$, respectively. The stress-strain curves under different magnetic flux densities had the same strain-hardening characteristic.

The dependence of the stiffness parameter of the first-order Ogden model on the magnetic flux density was analyzed, and a magnetic-field-dependent function of the stiffness parameter was proposed based on a magnetic dipole model. By combining the first-order Ogden hyperelastic model, the magnetic-field-dependent function of the stiffness parameter and the magnetization model of MRE, a new magneto-hyperelastic model was established. By comparing the model predictions with the experimental results, it was found that the proposed model could accurately predict the compressive stresses of the isotropic MRE within the tested magnetic flux density and strain ranges. The advantage of this magneto-hyperelastic model is that the magnetic-field-dependent compression property of the isotropic MRE can be predicted using only three model parameters, and a constant parameter $\mathrm{K}$ that represents the dependence of material stiffness on the magnetization of MRE is defined and can be easily determined. However, the proposed magneto-hyperelastic model overestimates the stresses for the strain over 0.3 due to the lack of consideration of the effects of the microstructure damage and the reconstruction of particle distribution within MRE material under large deformation.

Author Contributions: Conceptualization, Y.Q. and J.Z.; methodology, Y.Q. and J.Z.; validation, Y.Q., J.Z. and M.Z.; investigation, M.Z.; resources, J.Z.; data curation, Y.Q.; writing—original draft preparation, Y.Q.; writing一review and editing, J.Z.; supervision, L.L. and P.Z.; project administration, J.Z.; funding acquisition, J.Z. All authors have read and agreed to the published version of the manuscript.

Funding: This work was supported by the National Natural Science Foundation of China (NSFC), under Grant No.11202154, and by the Fundamental Research Funds for the Central Universities of China, under Grant Nos. WUT-2019-zy-233 and WUT-2020III027GX. 
Conflicts of Interest: The authors declare no conflict of interest.

\section{References}

1. Jolly, M.R.; Carlson, J.D.; Munoz, B.C. A model of the behaviour of magnetorheological materials. Smart Mater. Struct. 1996, 5, 607-614. [CrossRef]

2. Davis, L.C. Model of magnetorheological elastomers. J. Appl. Phys. 1999, 85, 3348-3351. [CrossRef]

3. Varga, Z.; Filipcsei, G.; Zrinyi, M. Magnetic field sensitive functional elastomers with tuneable elastic modulus. Polymer 2006, 47, 227-233. [CrossRef]

4. Soriahernandez, C.G.; Palaciospineda, L.M.; Eliaszuniga, A.; Peralesmartinez, A.; Martinezromero, O. Investigation of the Effect of Carbonyl Iron Micro-Particles on the Mechanical and Rheological Properties of Isotropic and Anisotropic MREs: Constitutive Magneto-Mechanical Material Model. Polymers 2019, 11, 1705. [CrossRef] [PubMed]

5. Schubert, G.; Harrison, P.G. Large-strain behaviour of magneto-rheological elastomers tested under uniaxial compression and tension, and pure shear deformations. Polym. Test. 2015, 42, 122-134. [CrossRef]

6. Deng, H.; Gong, X.; Wang, L. Development of an adaptive tuned vibration absorber with magnetorheological elastomer. Smart Mater. Struct. 2006, 15, N111-N116. [CrossRef]

7. Fu, J.; Lai, J.; Yang, Z.; Bai, J.; Yu, M. Fuzzy-neural network control for a magnetorheological elastomer vibration isolation system. Smart Mater. Struct. 2020, 29, 074001. [CrossRef]

8. Hu, T.; Xuan, S.; Ding, L.; Gong, X. Stretchable and magneto-sensitive strain sensor based on silver nanowire-polyurethane sponge enhanced magnetorheological elastomer. Mater. Des. 2018, 156, 528-537. [CrossRef]

9. Sedlacik, M.; Mrlik, M.; Babayan, V.; Pavlinek, V. Magnetorheological elastomers with efficient electromagnetic shielding. Compos. Struct. 2016, 135, 199-204. [CrossRef]

10. Yun, G.; Tang, S.; Sun, S.; Yuan, D.; Zhao, Q.; Deng, L.; Yan, S.; Du, H.; Dickey, M.D.; Li, W. Liquid metal-filled magnetorheological elastomer with positive piezoconductivity. Nat. Commun. 2019, 10, 1-9. [CrossRef]

11. Gordaninejad, F.; Wang, X.; Mysore, P. Behavior of thick magnetorheological elastomers. J. Intell. Mater. Syst. Struct. 2012, 23, 1033-1039. [CrossRef]

12. Ivaneyko, D.; Toshchevikov, V.; Saphiannikova, M.; Heinrich, G. Effects of particle distribution on mechanical properties of magneto-sensitive elastomers in a homogeneous magnetic field. Condens. Matter Phys. 2012, 15, 33601. [CrossRef]

13. Liao, G.; Gong, X.; Xuan, S.; Guo, C.; Zong, L. Magnetic-field-induced normal force of magnetorheological elastomer under compression status. Ind. Eng. Chem. Res. 2012, 51, 3322-3328. [CrossRef]

14. Koo, J.; Khan, F.; Jang, D.; Jung, H. Dynamic characterization and modeling of magneto-rheological elastomers under compressive loadings. Smart Mater. Struct. 2010, 19, 117002. [CrossRef]

15. Liao, G.; Gong, X.; Xuan, S. Magnetic field-induced compressive property of magnetorheological elastomer under high strain rate. Ind. Eng. Chem. Res. 2013, 52, 8445-8453. [CrossRef]

16. Vatandoost, H.; Norouzi, M.; Alehashem, S.M.; Smoukov, S.K. A novel phenomenological model for dynamic behavior of magnetorheological elastomers in tension-compression mode. Smart Mater. Struct. 2017, 26, 065011. [CrossRef]

17. Khanouki, M.A.; Sedaghati, R.; Hemmatian, M. Experimental characterization and microscale modeling of isotropic and anisotropic magnetorheological elastomers. Compos. Part B Eng. 2019, 176, 107311. [CrossRef]

18. Nadzharyan, T.A.; Sorokin, V.V.; Stepanov, G.V.; Bogolyubov, A.N.; Kramarenko, E.Y. A fractional calculus approach to modeling rheological behavior of soft magnetic elastomers. Polymer 2016, 92, 179-188. [CrossRef]

19. Liu, T.; Gong, X.; Xu, Y.; Xuan, S.; Jiang, W. Simulation of magneto-induced rearrangeable microstructures of magnetorheological plastomers. Soft Matter 2013, 9, 10069-10080. [CrossRef]

20. Wang, Q.; Dong, X.; Li, L.; Ou, J. A nonlinear model of magnetorheological elastomer with wide amplitude range and variable frequencies. Smart Mater. Struct. 2017, 26, 065010. [CrossRef]

21. Li, R.; Sun, L.Z. Viscoelastic responses of silicone-rubber-based magnetorheological elastomers under compressive and shear loadings. J. Eng. Mater. Technol. Trans. ASME 2013, 135, 021008. [CrossRef]

22. Ogden, R.W. Large deformation isotropic elasticity-on the correlation of theory and experiment for incompressible rubberlike solids. Proc. R. Soc. A Math. Phys. Eng. Sci. 1972, 326, 565-584. 
23. Sasso, M.; Palmieri, G.; Chiappini, G.; Amodio, D. Characterization of hyperelastic rubber-like materials by biaxial and uniaxial stretching tests based on optical methods. Polym. Test. 2008, 27, 995-1004. [CrossRef]

24. Li, W.; Xin, Z. Flexural fatigue life prediction of a tooth V-belt made of fiber reinforced rubber. Int. J. Fatigue 2018, 111, 269-277. [CrossRef]

25. Beda, T. Optimizing the Ogden strain energy expression of rubber materials. J. Eng. Mater. Technol. Trans. ASME 2005, 127, 351-353. [CrossRef]

26. Ivaneyko, D.; Toshchevikov, V.; Saphiannikova, M.; Heinrich, G. Magneto-sensitive Elastomers in a Homogeneous Magnetic Field: A Regular Rectangular Lattice Model. Macromol. Theory Simul. 2011, 20, 411-424. [CrossRef]

27. Ivaneyko, D.; Toshchevikov, V.; Borin, D.; Saphiannikova, M.; Heinrich, G. Mechanical Properties of Magneto-Sensitive Elastomers in a Homogeneous Magnetic Field: Theory and Experiment. Macromol. Symp. 2014, 338, 96-107. [CrossRef]

28. Fan, Y.C.; Gong, X.L.; Jiang, W.Q.; Zhang, W.; Wei, B.; Li, W.H. Effect of maleic anhydride on the damping property of magnetorheological elastomers. Smart Mater. Struct. 2010, 19, 055015. [CrossRef]

29. Farshad, M.; Le Roux, M. Compression properties of magnetostrictive polymer composite gels. Polym. Test. 2005, 24, 163-168. [CrossRef]

Publisher's Note: MDPI stays neutral with regard to jurisdictional claims in published maps and institutional affiliations.

(C) 2020 by the authors. Licensee MDPI, Basel, Switzerland. This article is an open access article distributed under the terms and conditions of the Creative Commons Attribution (CC BY) license (http://creativecommons.org/licenses/by/4.0/). 